\title{
Recent Advances on Imaging Porous Frameworks by Electron Microscopy Methods
}

\author{
Alvaro Mayoral ${ }^{1}$, Isabel Diaz ${ }^{2}$ and Jennifer E. Readman ${ }^{3}$ \\ 1. Advanced Microscopy Laboratory (LMA), Nanoscience Institute of Aragon (INA), University of \\ Zaragoza, Zaragoza, Spain. \\ 2. Instituto de Catálisis y Petroleoquimica (ICP), CSIC, Marie Curie, 2, 28049, Madrid, Spain \\ 3. School of Physical Sciences and Computing University of Central Lancashire, Preston, UK.
}

Zeolites are undoubtedly one of the most significant inorganic materials in the current industry due to their high importance in a variety of industries in the areas of catalysis, petrochemistry or detergents. Their structure can be briefly described as tetrahedra sharing corners and such importance has driven zeolite science to extensively grow since their initial discovery in 1756 . New synthetic mechanisms together with more sophisticated characterization methods are continuously being developed giving as a result over 200 different topologies. Among the several techniques for the analysis of these materials, electron microscopy methods are of particular interest due to the unique information that can be extracted, especially through transmission electron microscopy (TEM) combining imaging, diffraction and chemical analysis.

Recently, TEM has experienced a huge improvement with the implementation of the spherical aberration correctors which allow reaching sub-angstrom resolution in conventional microscopes. This revolutionary method of analysis has revealed the mater as it has never been observed showing with atomic exactitude the atomic conformation of all kind of materials [1]. With this type of equipment lateral resolution is only limited by the sample itself, which has to be thin enough for the electrons to be transfered but at the same time the material has to be sufficiently robust for not being affected by the electron beam. Unfortunately, it is known that molecular sieves suffer severely from beam damage which is associated to a radiolytic mechanism [2]. This effect has strongly limited the use of electron microscopy to study zeolites, zeotypes or metal organic frameworks (MOFs) and controlling the dose has been critical in order to acquire images of the structure [3]. Although traditionally electron microscopy observations have been performed in conventional TEM mode, this is using a parallel beam; the combination of the spherical correctors with the convergence beam, (Scanning Transmission Electron Microscopy) STEM mode, and a High Angle Annular Dark Field (HAADF) detector seems to be a more convenient approach due to its sensitivity to the atomic number.

Taking all this parameters into account it is possible to visualize the atomic disposition of ordered porous materials as it is the case of the titanosilicate ETS-10 [4]. Figure 1s shows the schematic representation of ETS-10 where the porous structure is orientated along the [110] with the Ti atoms represented in light blue, Si in dark blue and O in red. Due to the "Z" sensitivity it is possible to identify certain Ti columns in the experimental image, Figure $1 \mathrm{~b}$, as the brightest spots. Furthermore, this technique gains even more interest when heavier cations are introduced into the structure. With the intention of creating a material with luminescent properties, rare earth cations $(\mathrm{Eu}, \mathrm{Tb}$ and $\mathrm{Gd})$ where introduced into the parental ETS-10 containing $\mathrm{Na}$ and $\mathrm{K}$ as counterions [5]. These new materials were subjected to study by $\mathrm{C}_{\mathrm{s}}$-corrected STEM analyzing the new contrast observed in the material especially along the [110], parallel to the pores. Figure 2 displays the same image of the Eu loaded ETS-10 recorded with two different detectors at the same time which allows the visualization of the defects 
(Figure 2a) as well as a strongest contrast owed to the presence of Eu cations (Figure 2b). The results obtained here, not only allowed evaluating the ion-exchange capacity of a porous network but it also helped to understand the location of the initial light cations present in the material.

As a summary, despite all difficulties of beam sensitive materials modern aberration corrected electron microscopy methods can give invaluable information of structural features in ordered porous architectures. The frameworks can now be visualized in such a good quality that novel information at atomic level can be extracted helping to rationalize the design of new methods for the synthesis of zeolites and zeotypes. In addition, this methodology can be exported into the MOF field which is a significant more unstable material [6].

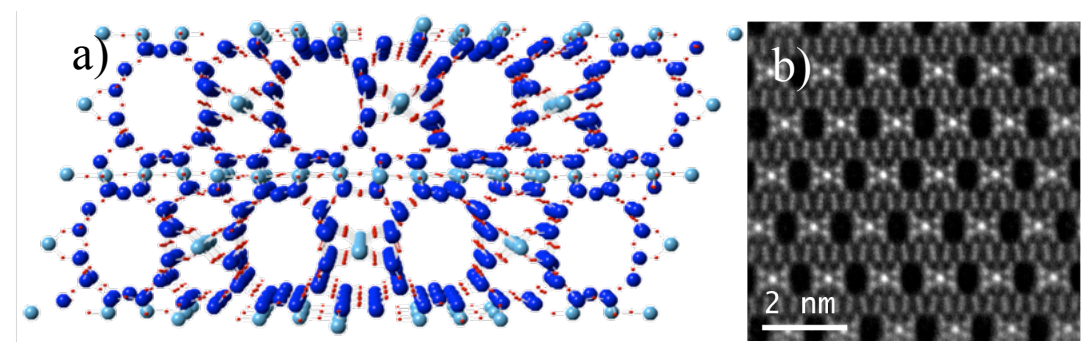

Figure 1. a) Schematic representation of ETS-10. b) $\mathrm{C}_{\mathrm{S}}$-corrected STEM HAADF image along the $[110]$.

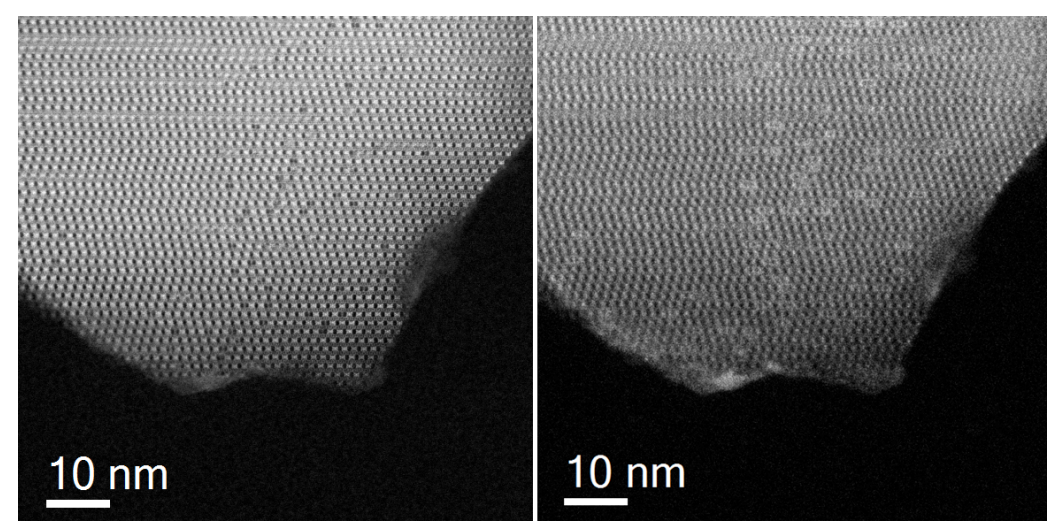

Figure 2. $\mathrm{C}_{\mathrm{s}}$-corrected STEM images of Eu-loaded ETS-10 a) showing the defects (so called double pores) and b) those defects cannot be observed; instead the strongest signal from Eu can be appreciated.

References:

[1] A Mayora et al, J. Mater. Chem. 21 (2010) 893-898.

[2] I. Díaz, A. Mayoral, Micron 42 (2011) 512-527.

[3] A. Mayoral et al, Micropor. Mesopor. Mater. 166 (2013) 117-122.

[4] A. Mayoral et al, Chem. Cat. Chem. 5 (2013) 2595-2598.

[5] A. Mayoral et al, Angew. Chem. Int. Ed. 55 (2016) 16127-16131.

[6] The research leading to these results has received funding from the European Union Seventh Framework Programme under Grant Agreement 312483, ESTEEM2 (Integrated Infrastructure InitiativeI3). 